\title{
Fasted plasma asprosin concentrations are associated with menstrual cycle phase, oral contraceptive use and training status in healthy women
}

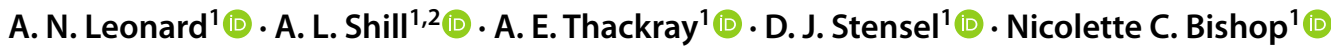

Received: 21 May 2020 / Accepted: 21 November 2020 / Published online: 8 December 2020

(C) The Author(s) 2020

\begin{abstract}
Purpose Asprosin, an orexigenic hormone that stimulates hepatic glucose release, is elevated in insulin resistance and associated with obesity. Plasma asprosin concentrations may also be related to female sex hormone levels; higher levels are reported in women with polycystic ovary syndrome (PCOS) but this may be related to peripheral insulin resistance also associated with PCOS. Clarification of female-specific factors influence on the plasma asprosin response is crucial for studies investigating asprosin. Therefore, this study determined the association of menstrual phase, oral contraceptive (OC) use (as a pharmacological influence on sex hormone levels) and training status (as a physiological influence on sex hormone levels) on plasma asprosin levels in pre-menopausal women.

Methods Fasting plasma asprosin, $17 \beta$-estradiol (E2) and progesterone, were assessed in 32 healthy untrained and trained women with regular menstrual cycles (non-OC; $n=8$ untrained, $n=6$ trained) or using OC ( $n=10$ untrained, $n=8$ trained) during early follicular, late follicular and mid-luteal menstrual phases (or the time-period equivalent for OC users).

Results Asprosin was lower in OC $\left(0.75 \pm 0.38 \mathrm{ng} \mathrm{mL}^{-1}\right)$ than non-OC users $\left(1.00 \pm 0.37 \mathrm{ng} \mathrm{mL}^{-1} ; p=0.022\right)$. Across a cycle, asprosin was highest in the early follicular equivalent time-point in OC users $\left(0.87 \pm 0.37 \mathrm{ng} \mathrm{mL}^{-1}\right)$ but highest in the mid-luteal phase in non-OC users $\left(1.09 \pm 0.40 \mathrm{ng} \mathrm{mL}^{-1}\right)$. Asprosin concentrations varied more across a cycle in untrained than trained women, with higher concentrations in the early follicular phase compared to the late follicular and mid-luteal (training status-by-menstrual phase interaction $p=0.028$ ).

Conclusion These findings highlight the importance of considering OC use, menstrual cycle phase and to a lesser extent training status when investigating circulating asprosin concentrations in females.
\end{abstract}

Keywords Female $\cdot$ Pre-menopausal $\cdot$ Birth control $\cdot$ Appetite hormone $\cdot$ Exercise

\section{Abbreviations \\ E2 17 $\beta$-Estradiol \\ EF Early follicular \\ ELISA Enzyme-linked immunosorbent assay}

Communicated by Fabio Fischetti.

Supplementary Information The online version contains supplementary material available at https://doi.org/10.1007/s0042 1-020-04570-8.

Nicolette C. Bishop

N.C.Bishop@lboro.ac.uk

1 School of Sport, Exercise and Health Sciences, National Centre for Sport and Exercise Medicine, Loughborough University, Loughborough, UK

2 English Institute of Sport, Loughborough, UK

$\begin{array}{ll}\text { ES } & \text { Effect size } \\ \text { LF } & \text { Late follicular } \\ \text { ML } & \text { Mid-luteal } \\ \text { OC } & \text { Oral contraceptive } \\ \text { PCOS } & \text { Polycystic ovary syndrome } \\ \text { SD } & \text { Standard deviation } \\ \text { T } & \text { Trained } \\ \text { UT } & \text { Untrained }\end{array}$

\section{Introduction}

Asprosin is a newly reported orexigenic protein hormone that is secreted from white adipose tissue and encoded by the final two exons of the FBN1 gene (Romere et al. 2016). FBN1 codes for the common extracellular matrix protein profibrillin 1 and it is the final terminus of this protein that 
makes up the hormone asprosin (Muthu and Reinhardt 2020). In a fasted state asprosin is elevated in the circulation, driving glucose release through a protein kinase A dependent mechanism (Romere et al. 2016).

The association between asprosin and appetite has been recently proposed; mice containing mutations affecting exon 65 of the FBN1 gene display hypophagia and extreme leanness compared to litter mates (Romere et al. 2016; Duerrschmid et al. 2017). In addition, these animals are protected from diabetes and obesity when exposed to dietary stress. Administration of recombinant asprosin rescues the hypophagia phenotype confirming the association with appetite. Clinical presentations in humans caused by mutations in FBN1 affecting asprosin are also associated with low subcutaneous adipose tissue and hypophagia, with significantly lower levels of circulating asprosin in those with mutations (Romere et al. 2016; Duerrschmid et al. 2017). In contrast, individuals who are overweight or have obesity have plasma asprosin concentrations which are up to fourfold above those of individuals with a healthy BMI (Ugur and Aydin 2019). This association of asprosin with body composition, presumably exerted through the effects on appetite, makes asprosin a target of significant interest in controlling energy balance and, therefore, disease in individuals with diabetes and obesity (Yuan et al. 2020).

Although the association with appetite has been demonstrated, elevated asprosin has been associated with negative consequences of diabetes, polycystic ovary syndrome (PCOS) and obesity, notably factors relating to insulin resistance, but a direct cause and effect relationship has yet to be robustly demonstrated in humans (Yuan et al. 2020). As research focusing on the role of asprosin in these conditions progresses, mounting data are being collected from women. Several of these studies have presented potentially contradictory results, but menstrual cycle phase and oral contraceptive (OC) use were not always controlled for (Li et al. 2018; Chang et al. 2019).

Metabolic disease is prevalent in both women and men, yet women are often excluded from research due to the cyclic changes in progesterone and oestrogen across a menstrual cycle (Sims and Heather 2018). Over a typical 28-day cycle, day 1 is the first day of menses marking the start of the follicular phase. The follicular phase is hallmarked by a steady rise in oestrogen which peaks at the end of the follicular phase (day 14; Reed and Carr 2000). The luteal phase is characterised by a steady rise in progesterone that peaks in the mid-luteal phase and gradually falls by the end of the luteal phase (Reed and Carr 2000). In contrast, users of OC have an almost completely blunted hormonal cycle due to inhibition of oestrogen and progesterone via exogenous hormones (Sims and Heather 2018). While a range of contraceptives are available, OC were reported by the United Nations (United Nation Department of Economic and Social
Affairs Population Divison 2015) to be the most widely used form of contraceptive worldwide. Therefore, users form a large and distinct physiological group making it necessary to examine both OC users and non-users when examining female physiology (Sims and Heather 2018).

Female sex hormones are thought to play a role in metabolic processes such as insulin sensitivity and liver glucose release (Mauvais-Jarvis et al. 2013; Campbell and Febbraio 2002; Salpeter et al. 2006; Stubbins et al. 2012; Wilsterman et al. 2017). It is, therefore, essential to understand changes in circulating asprosin, a hormone thought to be involved in metabolism, over the menstrual cycle and in OC users so that reliable conclusions can be made in future research into asprosin.

While OC use presents a pharmacological influence on circulating sex hormone levels, physical training status provides a physiological influence on sex hormone levels. It is not uncommon for active women to have reduced oestrogen and progesterone release (De Souza 2003). With regard to asprosin, an 8 week aerobic running program in rats with type 1 diabetes elicited a lowering of hepatic asprosin concentrations (Ko et al. 2019). Studies in women are limited, yet one study reports increases in non-fasted circulating asprosin concentrations in healthy active women with normal menstrual cycles after a bout of exercise during the midfollicular phase (Wiecek et al. 2018).

Therefore, the aim of this study was to determine the associations of menstrual phase, OC use (as a pharmacological influence on sex hormone levels) and training status (as a physiological influence on sex hormone levels) on plasma asprosin levels in pre-menopausal women. The findings of the study will help identify factors that need to be considered when assessing and interpreting asprosin data in females.

\section{Methods}

\section{Participants}

Healthy women $(n=32)$ with regular menstrual cycles or taking OC medication, for a minimum of three months, volunteered to participate in this observational study (Table 1). The women were untrained OC users (OC; $n=10)$, untrained non-OC users (non-OC; $n=8$ ), trained oral contraceptive users (OC; $n=8)$ or trained non-oral contraceptive users (non-OC; $n=6$ ). Trained was defined as participating in $\geq 3 \mathrm{~h}$ of structured purposeful exercise (e.g. team training or organised sessions) per week as determined by a selfreported training log. Trained and untrained women reported exercising 201-825 and 0-180 min per week, respectively. Trained women consisted of highly trained team sports athletes $(n=6)$, a recreationally trained team sports athlete $(n=1)$, highly trained endurance athletes $(n=4)$, a 
Table 1 Participant characteristics in untrained and trained groups who use oral contraceptives (OC) or do not use OCs (non-OC). All values are presented as mean $\pm \mathrm{SD}$

\begin{tabular}{|c|c|c|c|c|}
\hline & \multicolumn{2}{|l|}{ Untrained } & \multicolumn{2}{|l|}{ Trained } \\
\hline & $\mathrm{OC}(n=10)$ & Non-OC $(n=8)$ & $\mathrm{OC}(n=8)$ & Non-OC $(n=6)$ \\
\hline Age (years) & $24 \pm 3$ & $27 \pm 5$ & $24 \pm 2$ & $25 \pm 5$ \\
\hline Height (cm) & $168.0 \pm 7.5$ & $169 \pm 6.2$ & $167.8 \pm 5.1$ & $166 \pm 4.1$ \\
\hline Body mass (kg) & $63.1 \pm 10.5$ & $62.6 \pm 7.6$ & $62.1 \pm 9.9$ & $61.1 \pm 7.3$ \\
\hline BMI $\left(\mathrm{kg} / \mathrm{m}^{2}\right)$ & $22.3 \pm 2.8$ & $21.9 \pm 1.9$ & $21.9 \pm 2.7$ & $22.2 \pm 2.4$ \\
\hline Exercising minutes (mean min week ${ }^{-1}$ ) & $78 \pm 52^{*}$ & $71 \pm 70^{*}$ & $408 \pm 204$ & $382 \pm 64$ \\
\hline Menstrual cycle length (days) & $28 \pm 0$ & $27 \pm 2$ & $28 \pm 1$ & $31 \pm 6$ \\
\hline Day ovulation detected & - & $13 \pm 2$ & - & $16 \pm 1$ \\
\hline
\end{tabular}

*Main effect of training status $p \leq 0.001$ recreationally trained endurance athlete $(n=1)$, a highly trained middle-distance athlete $(n=1)$ and a highly trained power lifter $(n=1)$. Untrained women reported doing casual exercise such as going to the gym, attending exercise classes, running, walking, cycling and or no organised exercise at all.

$\mathrm{OC}$ use was defined as using a monophasic $\mathrm{OC}$ for at least three months. A non-user (non-OC) was defined as no use of hormonal contraceptives for at least three months. Untrained OC reported taking: combined levonorgestrel $(0.15 \mathrm{mg})$ and ethinylestradiol $(0.03 \mathrm{mg} ; n=6)$; combined norethisterone acetate $(1.5 \mathrm{mg})$ and ethinylestradiol $(0.03 \mathrm{mg} ; n=1)$; combined desogestrel $(0.15 \mathrm{mg})$ and ethinylestradiol $(0.03 \mathrm{mg}$; $n=1)$; combined norgestimate $(0.25 \mathrm{mg})$ and ethinylestradiol $(0.035 \mathrm{mg} ; n=1)$; or norethisterone $(0.35 \mathrm{mg} ; n=1)$. Trained OC reported taking: combined levonorgestrel $(0.15 \mathrm{mg})$ and ethinylestradiol $(0.03 \mathrm{mg} ; n=3)$; combined norethisterone acetate $(1.0 \mathrm{mg})$ and ethinylestradiol $(0.03 \mathrm{mg} ; n=2)$; combined desogestrel $(0.15 \mathrm{mg})$ and ethinylestradiol $(0.03 \mathrm{mg}$; $n=1)$; combined cyproterone acetate $(2.0 \mathrm{mg})$ and ethinylestradiol $(0.035 \mathrm{mg} ; n=1)$; or combined dienogest $(2.0 \mathrm{mg})$ and ethinylestradiol $(0.03 \mathrm{mg} ; n=1)$.

All participants completed a health-screen questionnaire and provided written informed consent to participate, after being informed of the rationale and protocol of the study. Further health-screen questionnaires and a willingness to participate form were completed at the beginning of each laboratory visit. The study protocol was approved by the local University Ethical Committee. In a preliminary visit a menstrual cycle questionnaire was completed to estimate cycle length and the following exclusion criteria were assessed: illnesses in the three weeks prior to laboratory testing; evidence of kidney disease or history of cardiovascular disease or metabolic disease; high blood pressure or dyslipidaemia; smokers or users of medical or illegal drugs that affect digestion, metabolism or inflammation; dieting or reported extreme dietary habits.

\section{Main visits}

For non-OC users, the three laboratory visits were scheduled to capture the early follicular (first 6 days), late follicular (days 9-13) and mid-luteal (days 19-23) menstrual phases of a single menstrual cycle (Fig. 1). In OC users, these timepoints refer to the time period equivalent to the cycle phases in non-OC users. These were estimated based on a 28 -day cycle with day 1 being the first day of menses or, for OC users, the first day without an OC dose resulting in a withdrawal bleed (Fig. 1). Participants were given commercially available ovulation midstream urine test sticks (Onestep, manufactured by AI DE Diagnostic Co. Ltd., China) to use for an eight-day period spanning the predicted ovulation day, commencing monitoring 3 days prior to the predicted
Fig. 1 Menstrual cycle phases, or timepoint equivalents (for oral contraceptive users), when main visits occurred, based on a typical 28-day menstrual cycle, where fasted blood samples were taken and analysed for circulating concentrations of asprosin, 17 $\beta$-estradiol and progesterone in healthy women

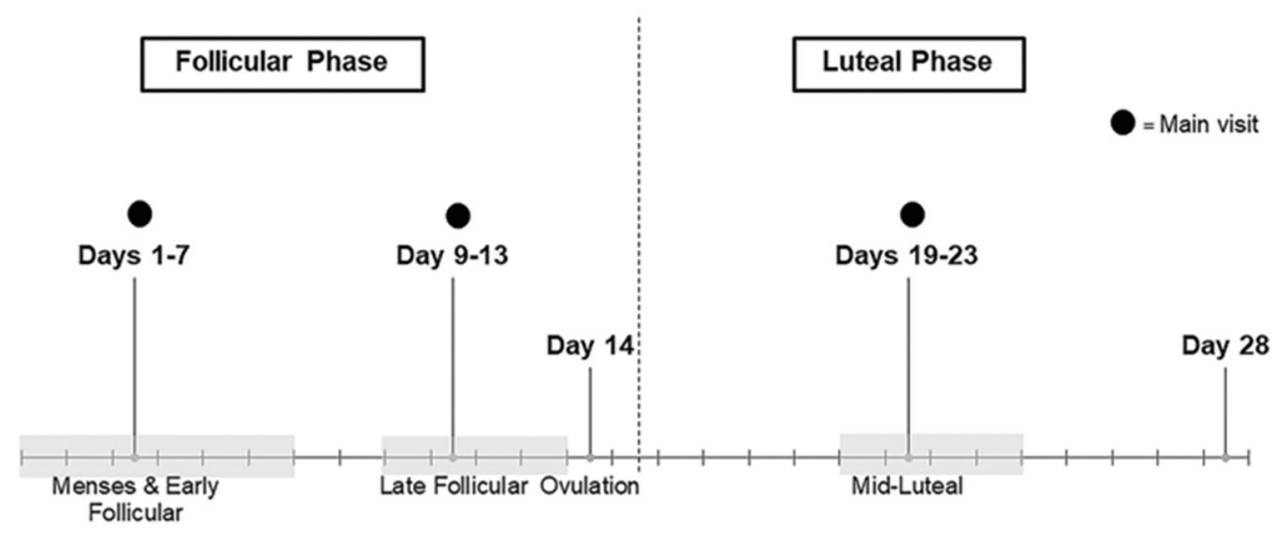

Springer 
ovulation day, following the manufacturer's instructions. The results of each test were recorded on the participants' diaries.

All laboratory visits began in the morning between 07:00 and 09:00 after an overnight fast of at least $10 \mathrm{~h}$. Upon arrival, participants stature and body mass were measured followed by $15 \mathrm{~min}$ of seated rest before a venous blood sample was taken from an antecubital vein. After the final laboratory visit, participants contacted a member of the research team when menses next occurred to determine the total length of their cycle.

\section{Plasma analysis}

Whole blood samples were collected into one $2.6 \mathrm{~mL}$ Lithium Heparin (16 IU heparin $\mathrm{mL}^{-1}$ ) and one $9 \mathrm{~mL}$ EDTA (1.6 mg mL ${ }^{-1}$; S-Monovette, Sarsdedt, Leicester UK) tube for female sex hormone and asprosin analyses, respectively. Blood was centrifuged at $4{ }^{\circ} \mathrm{C}$ for $10 \mathrm{~min}$ at $3500 \mathrm{rpm}$ and plasma was extracted and frozen at $-80^{\circ} \mathrm{C}$ for subsequent analysis.

Asprosin $\left(\mathrm{ng} \mathrm{mL}^{-1}\right)$ was determined in EDTA plasma using a commercially available enzyme-linked immunosorbent assay (ELISA), as per the manufacturer's instructions (Abbexa, catalogue no. abx257694; Abbexa, Cambridge, UK). The within assay co-efficient of variation was $12.4 \%$.

Heparinised plasma was assessed for $17 \beta$-estradiol (E2; pg $\mathrm{mL}^{-1}$ ) and progesterone ( $\mathrm{ng} \mathrm{mL}^{-1}$ ) using commercially available ELISAs, as per the manufacturer's instructions (IBL International, Hamburg, Germany). The within assay co-efficient of variation for the duplicate analyses was $1.4 \%$ for $\mathrm{E} 2$ and $1.3 \%$ for progesterone.

\section{Statistical analyses}

An a priori power calculation was conducted using $\mathrm{G}^{*}$ Power 3.1.9.2 (Faul et al. 2007) based on the mean difference (and SD of the difference) in oestrogen concentrations between early and late follicular phases (given the rationale that asprosin would be influenced by menstrual phase and oestrogen levels). It was calculated that 24 participants ( 6 per group) would have $>95 \%$ power at the 0.05 level to detect a Cohen's $d_{z}$ of 1.77 .

Using the statistical software SPSS v.24 (IBM Corporation) linear mixed models were used to examine differences in asprosin, E2 and progesterone concentration and body mass with fixed effects modelled of OC use (OC or nonOC), menstrual phase (early follicular, late follicular and mid-luteal) and training status (trained or untrained). Models were performed with repeated measures on the menstrual cycle variable. Where statistically significant main effects and interactions were identified, post-hoc analysis was performed using Fisher's least significant difference.
Residuals were used to check for normality and distribution. Non-normally distributed data (E2 and progesterone) were logged, and models were run on the logged data. Absolute standardised effect sizes (ES) were calculated to supplement important findings using the descriptors outlined by Cohen (1988): $<0.2$ trivial, $0.2-<0.5$ small, $0.5-<0.8$ moderate and $\geq 0.8$ large. To retain clarity and interpretation, the data is displayed graphically to present the statistically significant main and interaction effects. This means that figures only depict significant group interactions (e.g. a fixed effect for the interaction between OC use and menstrual phase on plasma asprosin concentration does not include training status as a grouping variable when running the model and thus the figure would not include training status as an aspect for grouping means on the graph). However, individual data are displayed by OC use and training status in the Electronic Supplementary Material for transparency, but the means have been modelled and were not statistically significant when grouped as they are in the supplementary figures.

Pearson correlation coefficients were quantified to examine relationships between asprosin and sex hormones and any association with OC use. Thresholds of $0.1,0.3$ and 0.5 were used to define Pearson correlation coefficients as small, moderate and large, respectively (Cohen 1988). All data is reported as the mean \pm standard deviation (SD). Statistical significance was accepted as $p<0.05 .95 \%$ confidence intervals (CI) for the mean absolute difference between groups were calculated.

\section{Results}

\section{OC vs. non-OC}

Figure 2 displays asprosin concentrations across menstrual cycle phases in OC and non-OC users. Non-OC users had higher asprosin levels compared to OC users (main effect OC use $p=0.022 ; \mathrm{ES}=0.66$ (moderate effect); $95 \% \mathrm{CI}-0.51$, $-0.04 \mathrm{ng} \mathrm{mL}^{-1}$ ). Linear mixed models also showed an interaction between OC use and menstrual phase $(p=0.011)$. Post hoc analysis revealed that $\mathrm{OC}$ users had higher asprosin concentrations in early follicular compared to late follicular $(p=0.032 ; \mathrm{ES}=0.46$ (small-to-moderate effect); 95\% CI $\left.0.01,0.30 \mathrm{ng} \mathrm{mL}^{-1}\right)$ and mid-luteal $(p=0.014 ; \mathrm{ES}=0.53$ (moderate effect); 95\% CI 0.04, $0.33 \mathrm{ng} \mathrm{mL}^{-1}$ ) phases whereas non-OC users had higher asprosin concentrations in mid-luteal compared to late follicular phase ( $p=0.048$; $\mathrm{ES}=0.38$ (small effect); $95 \% \mathrm{CI} 0.001,0.33 \mathrm{ng} \mathrm{mL}^{-1}$ ). For non-OC users, the $95 \% \mathrm{CI}$ for the difference in asprosin concentration between the early follicular and the mid-luteal phase overlapped zero, and the standardised ES was small $\left(p=0.063 ; \mathrm{ES}=0.36 ; 95 \% \mathrm{CI}-0.01,0.32 \mathrm{ng} \mathrm{mL}^{-1}\right)$. 


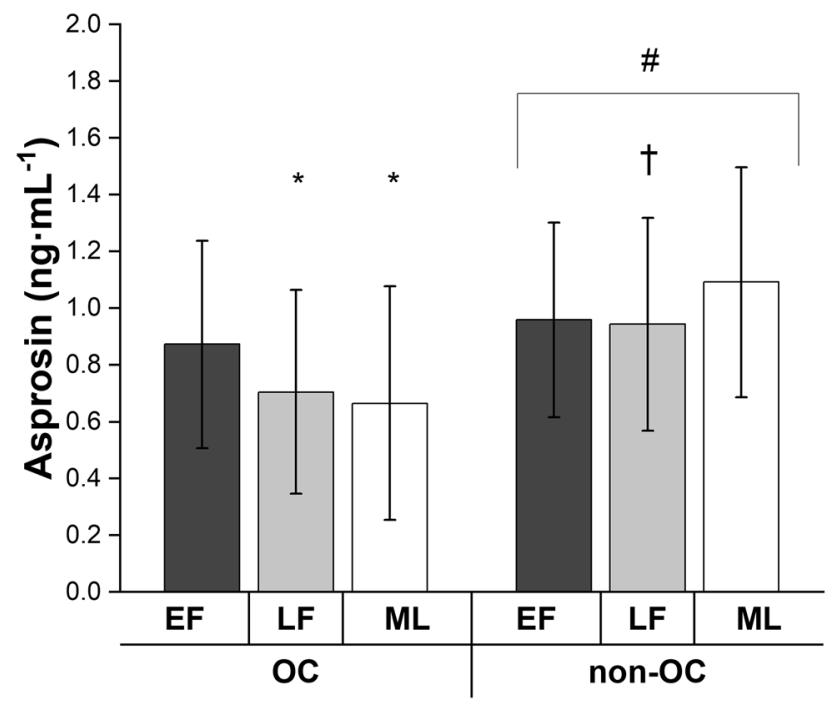

Fig. 2 Fasted circulating plasma asprosin concentrations in oral contraceptive (OC) users and non-users (non-OC) in the early follicular (EF; first 6 days), late follicular (LF; days 9-13) and mid-luteal (ML; days 19-23) mensural cycle phases (or time point equivalent for OC users). ${ }^{\dagger}$ Significantly lower than ML, within non-OC; *significantly lower than $\mathrm{EF}$, within $\mathrm{OC}$; " significantly higher compared to OC; $p<0.05$

Asprosin positively correlated with progesterone $(p=0.019)$ and E2 $(p=0.009)$ among all 32 participants in the mid-luteal phase (Table 2). However, when the data were split by OC use the positive correlation of asprosin with progesterone was only seen in OC users ( $p=0.001$; Table 2$)$.

Figure 3 displays progesterone and E2 concentrations across menstrual cycle phases in OC and non-OC users. Linear mixed models also showed an interaction between $\mathrm{OC}$ use and menstrual phase for progesterone and E2 $(p \leq 0.046)$. Post hoc analysis revealed that progesterone and E2 values were similar across the menstrual cycle in OC users
( $p \geq 0.142 ; \mathrm{ES} \leq 0.32$ (small effect). In non-OC user's, progesterone was higher in mid-luteal compared to early follicular $(p<0.0001 ; \mathrm{ES}=2.72$ (large effect); 95\% CI 9.22, $\left.13.22 \mathrm{ng} \mathrm{mL}^{-1}\right)$ and late follicular $(p<0.0001 ; \mathrm{ES}=2.69$ (large effect); 95\% CI 9.20, $13.21 \mathrm{ng} \mathrm{mL}^{-1}$ ) phases whereas E2 was lower in the early follicular compared to late follicular $(p<0.0001 ; \mathrm{ES}=1.21$ (large effect) 95\% CI - 106.17, $\left.-30.86 \mathrm{pg} \mathrm{mL}^{-1}\right)$ and mid-luteal $(p<0.0001 ; \mathrm{ES}=1.39$ (large effect); 95\% CI $-89.59,-14.28 \mathrm{pg} \mathrm{mL}^{-1}$ ) phases.

Similar findings were apparent when the post-hoc analysis of the OC used by menstrual phase interactions were explored by comparing concentrations directly between groups at each cycle phase. Specifically, asprosin concentrations in the early follicular phase were not statistically different between OC $\left(0.87 \pm 0.37 \mathrm{ng} \mathrm{mL}^{-1}\right)$ and non-OC $\left(0.96 \pm 0.34 \mathrm{ng} \mathrm{mL}^{-1}\right)$ users $(p=0.379 ; \mathrm{ES}=0.24$ (small effect); $95 \%$ CI $-0.38,0.15)$. However, asprosin concentrations were higher in non-OC users than OC users in the late follicular (non-OC $=0.94 \pm 0.37 \mathrm{ng} \mathrm{mL}^{-1}$; $\mathrm{OC}=0.71 \pm 0.36 \mathrm{ng} \mathrm{mL}-1 ; p=0.05 ; \mathrm{ES}=0.65$ (moderate effect); $95 \% \mathrm{CI} 0.00,0.53)$ and mid-luteal (non$\mathrm{OC}=1.09 \pm 0.40 \mathrm{ng} \mathrm{mL}{ }^{-1} ; \mathrm{OC}=0.67 \pm 0.41 \mathrm{ng} \mathrm{mL}^{-1}$; $p=0.001 ; \mathrm{ES}=1.05$ (large effect); 95\% CI 0.19, 0.72) phases.

\section{Trained vs untrained}

Linear mixed models also showed an interaction between training status and menstrual phase on asprosin concentrations $(p=0.028)$. Post hoc analysis revealed that untrained, but not trained, women had significantly higher asprosin concentrations in the early follicular compared to late follicular $(p=0.045 ; \mathrm{ES}=0.44$ (small-to-moderate effect); 95\% CI 0.003, $\left.0.29 \mathrm{ng} \mathrm{mL}^{-1}\right)$ and mid-luteal $(p=0.023$; $\mathrm{ES}=0.49$ (small-to-moderate effect); $95 \%$ CI 0.02 , $0.31 \mathrm{ng} \mathrm{mL}{ }^{-1}$; Fig. 4) phases. Circulating concentrations

Table 2 Pearson correlation $(r)$ between plasma asprosin, oestrogen and progesterone in all women and women who use oral contraceptives (OC) or do not use OCs (non-OC) in the mid-luteal phase

\begin{tabular}{|c|c|c|c|c|c|c|}
\hline & & \multicolumn{5}{|c|}{ All women $(n=32)$} \\
\hline & & \multicolumn{2}{|l|}{ Asprosin } & \multicolumn{2}{|l|}{ Progesterone } & Oestrogen \\
\hline Asprosin & & \multicolumn{2}{|l|}{ - } & \multicolumn{2}{|l|}{$r=0.418^{\mathrm{a}}$} & $r=0.462^{b}$ \\
\hline \multirow[t]{3}{*}{ Progesterone } & & & & - & & $r=0.642^{b}$ \\
\hline & \multicolumn{3}{|c|}{$\mathrm{OC}(n=18)$} & \multicolumn{3}{|c|}{ Non-OC $(n=14)$} \\
\hline & Asprosin & Progesterone & Oestrogen & Asprosin & Progesterone & Oestrogen \\
\hline Asprosin & - & $r=0.735^{\mathrm{b}}$ & $r=0.383$ & - & $r=-0.008$ & $r=0.234$ \\
\hline Progesterone & & - & $r=0.523^{\mathrm{a}}$ & & - & $r=0.517$ \\
\hline
\end{tabular}

Thresholds of 0.1, 0.3 and 0.5 were used to define Pearson correlation coefficients as small, moderate and large, respectively (Cohen 1988)

${ }^{\mathrm{a}}$ Correlation is significant at the 0.05 level

${ }^{\mathrm{b}}$ Correlation is significant at the 0.01 level 

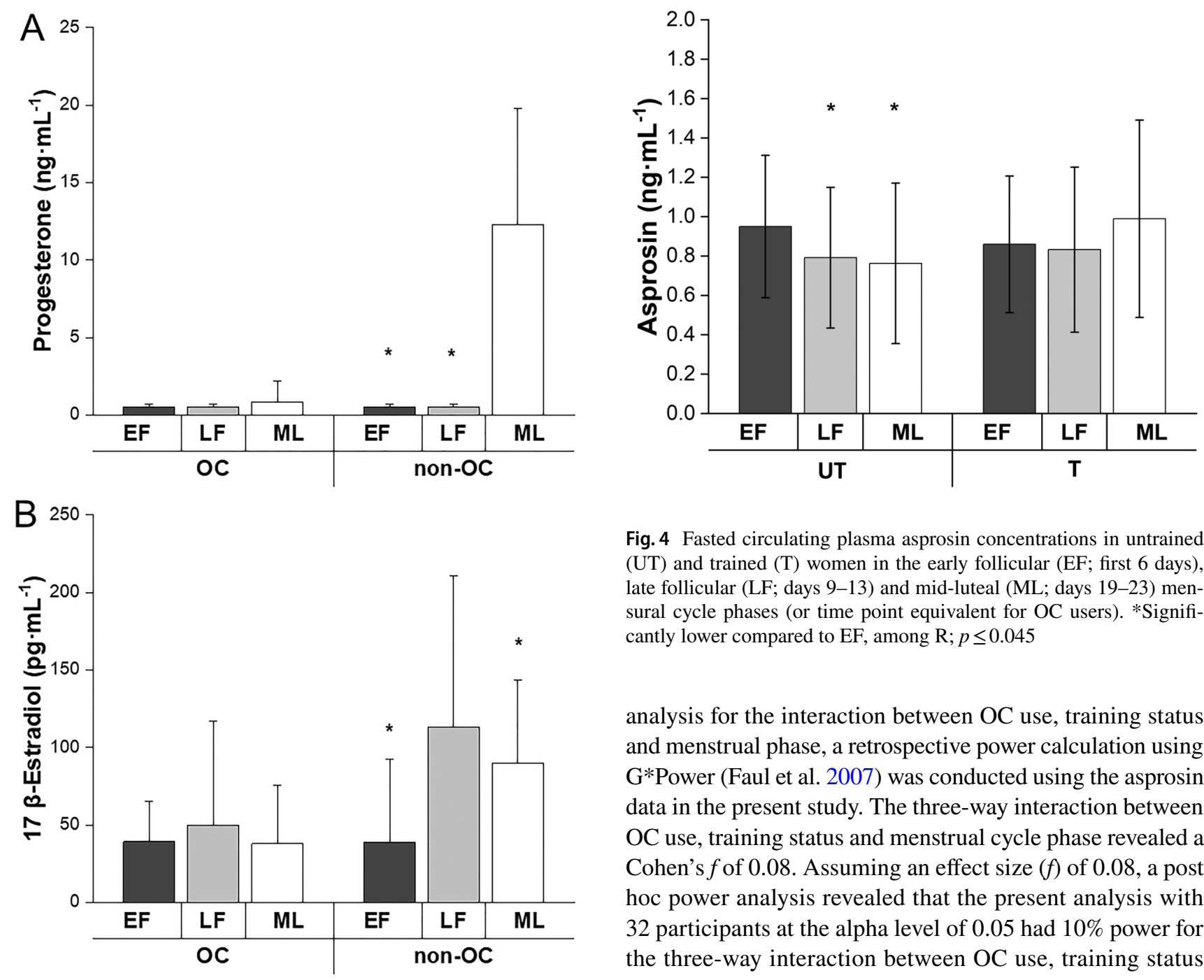

Fig. 4 Fasted circulating plasma asprosin concentrations in untrained (UT) and trained (T) women in the early follicular (EF; first 6 days), late follicular (LF; days 9-13) and mid-luteal (ML; days 19-23) mensural cycle phases (or time point equivalent for OC users). *Significantly lower compared to EF, among R; $p \leq 0.045$

analysis for the interaction between OC use, training status and menstrual phase, a retrospective power calculation using G*Power (Faul et al. 2007) was conducted using the asprosin data in the present study. The three-way interaction between OC use, training status and menstrual cycle phase revealed a Cohen's $f$ of 0.08 . Assuming an effect size $(f)$ of 0.08 , a post hoc power analysis revealed that the present analysis with 32 participants at the alpha level of 0.05 had $10 \%$ power for the three-way interaction between $\mathrm{OC}$ use, training status and menstrual cycle phase. Furthermore, it was estimated

Fig. 3 Fasted circulating plasma progesterone (a) and $17 \beta$-Estradiol (b) concentrations in oral contraceptive (OC) users and non-users (non-OC) in the early follicular (EF; first 6 days), late follicular (LF; days 9-13) and mid-luteal (ML; days 19-23) mensural cycle phases (or time point equivalent for OC users). *Significantly $(p \leq 0.001)$ lower hormone values in EF and LF versus ML (panel a) and in EF and ML versus LF (panel b) within non-OC users

of E2 and progesterone were similar between trained and untrained women [main effect of training status $p \geq 0.526$; $\mathrm{ES} \leq 0.06$ (trivial effect)].

\section{Three-way interaction between $O C$ use, training status and menstrual phase}

There were no statistically significant three-way interactions between OC use group, training status and menstrual cycle phase for asprosin, progesterone or E2 ( $p \geq 0.245$; see Figures in Electronic Supplementary Material). In the absence of existing asprosin data to perform an a priori power that a sample size of 380 ( 95 per group) participants would be required, for a three-way interaction, to detect the effect size $(f)$ of 0.08 with an alpha level of 0.05 and $80 \%$ power.

\section{Discussion}

The primary finding of the present study is that circulating fasted asprosin concentrations are associated with OC use and menstrual cycle phase in healthy young women. Importantly these findings suggest that OC use and menstrual phase should be controlled for when assessing and interpreting alterations in circulating asprosin levels. The present study reported that plasma asprosin: (1) is lower in OC users than in women with a normal menstrual cycle; (2) is highest in the early follicular equivalent time-point in OC users but highest in the mid-luteal phase in non-OC users, suggesting a potential phase-dependent change in plasma asprosin concentrations; and (3) demonstrated a phasic response across a menstrual cycle in untrained, but not highly trained, women. 
Of note, OC users had lower plasma asprosin concentrations compared with non-OC users, and levels were highest in $\mathrm{OC}$ users in the early follicular phase, yet highest in the mid-luteal phase in non-OC users. In addition, asprosin concentrations were not different between $\mathrm{OC}$ users and non-OC users in the early follicular phase, menses, when OC users were not taking the pill. However, compared to non-OC, the OC users asprosin concentrations decreased across the timepoints, with the lowest asprosin concentrations in the midluteal timepoint where OC users have been taking the pill for the longest amount of time. In the present study 'early follicular' was defined as days 1-6 which captured the pill-free week in OC users. Since OC users' asprosin concentrations were lower while taking OC (late follicular and mid-luteal) but higher in the pill-free week (early follicular) it is logical to infer that $\mathrm{OC}$ use is contributing to lower circulating plasma asprosin concentrations in women. OC users had $39 \%$ lower plasma asprosin compared to non-OC users in the mid-luteal phase. This difference is similar in magnitude to the difference in circulating plasma asprosin concentration in a nonfasted human to their fasted state (Romere et al. 2016) and emphasizes the need to control for OC use in the design of research studies assessing asprosin in women.

These findings suggest that OC use attenuates the natural phasic response of asprosin across the menstrual cycle. Amongst non-OC users, asprosin was highest in the midluteal compared to late follicular phase but asprosin midluteal concentrations were not significantly different from early follicular concentrations. This rise in asprosin concentration in the mid-luteal phase appears to be driven predominantly by trained women, although this is not statistically significant and, therefore, we cannot comment further on this trend (see electronic supplementary material Fig. 1). Across a typical menstrual cycle, the hallmark of the midluteal phase is a progesterone peak and plateau which occurs concomitantly with a smaller rise in oestrogen. However, the present study did not observe a correlation between asprosin and progesterone or E2 among non-OC users. This agrees with Chang et al (2019) who also did not see a correlation between E2 and asprosin in non-OC users. Furthermore, Li et al. (2018) also reported no correlation between asprosin and progesterone in women with PCOS, but did report a positive correlation between asprosin and E2 in normal weight (but not overweight) women with PCOS (Li et al. 2018). However, the latter study did not list $\mathrm{OC}$ use as an exclusion criterion for participants with PCOS. The present study did find a positive correlation between asprosin and progesterone among OC users only with the data was split by OC use and all participants in the present study were of normal weight. The inclusion of both OC users and non-OC users in the study by Li et al. (2018) could explain the disparity in findings and highlights the need to control for OC use when examining circulating asprosin concentrations in women.
Studies have found a positive correlation between testosterone and asprosin in women with PCOS (Li et al. 2018; Chang et al. 2019). In addition, Chang et al. (2019) reported a correlation between asprosin and follicle-stimulating hormone. Considering these studies involved women with diagnosed PCOS, a condition known to effect androgens, this could account for their findings (Goodarzi et al. 2011). It is possible that asprosin interacts with other sex hormones not measured in the present study, which may elevate asprosin concentrations in the mid-luteal phase. Therefore, further research is needed into circulating asprosin concentrations and sex hormones such as testosterone and follicle-stimulating hormone. In the meantime, it is prudent to account for menstrual cycle phase in the design of future studies until it is clear how asprosin is affected by sex hormones.

The present study demonstrated higher asprosin concentrations only amongst untrained women in the early follicular compared to late follicular and mid-luteal phases. The increased asprosin concentration in the early follicular phase appears to be driven predominantly by OC users, although this is not statistically significant and, therefore, we cannot comment further on this trend (see electronic supplementary material Fig. 1). This mirrors the increase in asprosin during the pill-free week observed the OC users, as the timing of the early follicular phase would coincide with the pill free week. In contrast, asprosin concentrations did not show a phasic response in trained women, irrespective of OC use, suggesting that training status does influence asprosin concentrations. As previously mentioned, testosterone and follicular stimulating hormone have been reported to correlate with asprosin concentrations in studies with less control on OC use and menstrual phase in females with PCOS ( $\mathrm{Li}$ et al. 2018; Chang et al. 2019). Since it is possible that some sex-related homones are correlated with asprosin it could be that hormones not measured in the present investigation were suppressed or increased in our trained women which prevented the natural cycling of asprosin concentrations to occur (Cho et al. 2017). Our findings also suggest training status may need to be considered when measuring novel biomarkers or variables known to be influenced by the menstrual cycle due to potential luteal phase anomalies that may affect results.

The findings of the present study are preliminary and require confirmation in future research. A priori power calculations based on meaningful changes in oestrogen along with our inclusion of effect sizes allow our results to be interpreted with more confidence. Although, to examine a specific three-way interaction between OC use, training status and menstrual cycle phase (see Figures in Electronic Supplementary Material) a future study with larger sample size is required, as demonstrated by our retrospective power analysis. That said, our calculations suggest a sample size of almost 400 women which may limit the 
feasibility of conducting such a study. However, given the difference in asprosin between OC user groups and the pattern of change in concentrations across the menstrual cycle was small-to-moderate in magnitude, it may be prudent to consider OC use when investigating asprosin responses in women. It should also be acknowledged that various types of monophasic OCs were used by women in the present study, all of which could exert different magnitude of effects on asprosin.

In conclusion, the present study demonstrated markedly lower plasma asprosin concentrations in OC users compared to women who are not taking OC and have a normal menstrual cycle. Furthermore, asprosin concentrations appear to be influenced by menstrual cycle phase in non-OC users and demonstrated greater variation across a cycle in untrained than trained women. Therefore, OC use, menstrual cycle phase and training status should be considered in the design of future studies assessing and interpreting asprosin data in females.

Acknowledgements This research was supported by the National Institute for Health Research NIHR Leicester Biomedical Research Centre. The views expressed are those of the authors and not necessarily those of the NHS, the NIHR or the Department of Health and Social Care.

Author contributions ANL, ALS and NCB conceived and designed the research. ANL and ALS conducted experiments. ANL, ALS and AET ran biochemical analysis on samples. ANL and AET ran statistical analysis on data. DJS and NCB provided expertise in the area. ANL prepared the manuscript. All authors read and approved the manuscript.

Funding This study was funded by the Biomedical Research Centre at Leicester, UK and Loughborough University, UK.

Availability of data and material The datasets generated during and/or analysed during the current study are available from the corresponding author on reasonable request. Relevant individual data are also included in the Electronic Supplementary Material.

Code availability Not applicable.

\section{Compliance with ethical standards}

Conflict of interest The authors declare that they have no conflict of interest.

Ethics approval All procedures performed in studies involving human participants were in accordance with the ethical standards of the institutional and/or national research committee and with the 1964 Helsinki Declaration and its later amendments or comparable ethical standards. The study was approved by the Loughborough University Ethics committee (R17-P077).

Consent to participate Informed consent was obtained from all individual participants included in the study.

Consent for publication Participants signed informed consent regarding publishing their data.
Open Access This article is licensed under a Creative Commons Attribution 4.0 International License, which permits use, sharing, adaptation, distribution and reproduction in any medium or format, as long as you give appropriate credit to the original author(s) and the source, provide a link to the Creative Commons licence, and indicate if changes were made. The images or other third party material in this article are included in the article's Creative Commons licence, unless indicated otherwise in a credit line to the material. If material is not included in the article's Creative Commons licence and your intended use is not permitted by statutory regulation or exceeds the permitted use, you will need to obtain permission directly from the copyright holder. To view a copy of this licence, visit http://creativecommons.org/licenses/by/4.0/.

\section{References}

Campbell SE, Febbraio MA (2002) Effect of the ovarian hormones on GLUT4 expression and contraction-stimulated glucose uptake. Am J Physiol Endocrinol Metab 282(5):E1139-E1146. https:// doi.org/10.1152/ajpendo.00184.2001

Chang CL, Huang SY, Hsu YC, Chin TH, Soong YK (2019) The serum level of irisin, but not asprosin, is abnormal in polycystic ovary syndrome patients. Sci Rep 9(1):6447. https://doi.org/10.1038/ s41598-019-42061-9

Cho GJ, Han SW, Shin J-H, Kim T (2017) Effects of intensive training on menstrual function and certain serum hormones and peptides related to the female reproductive system. Medicine 96(21):e6876. https://doi.org/10.1097/MD.0000000000006876

Cohen J (1988) Statistical power analysis for the behaviour sciences, 2nd edn. Routledge, London

De Souza MJ (2003) Menstrual disturbances in athletes: a focus on luteal phase defects. Med Sci Sports Exerc 35(9):1553-1563. https ://doi.org/10.1249/01.MSS.0000084530.31478.DF

Duerrschmid C, He Y, Wang C, Li C, Bournat JC, Romere C, Chopra AR et al (2017) Asprosin is a centrally acting orexigenic hormone. Nat Med 23(12):1444-1453. https://doi.org/10.1038/nm.4432

Faul F, Erdfelder E, Lang A-G, Buchner A (2007) G*Power 3: a flexible statistical power analysis program for the social, behavioral, and biomedical sciences. Behav Res Methods 39(2):175-191. https://doi.org/10.3758/BF03193146

Goodarzi MO, Dumesic DA, Chazenbalk G, Azziz R (2011) Polycystic ovary syndrome: etiology, pathogenesis and diagnosis. Nat Rev Endocrinol 7(4):219-231. https://doi.org/10.1038/nrend o.2010.217

Ko JR, Seo DY, Kim TN, Park SH, Kwak H-B, Ko KS, Han J et al (2019) Aerobic exercise training decreases hepatic asprosin in diabetic rats. J Clin Med 8(5):666. https://doi.org/10.3390/jcm80 50666

Li X, Liao M, Shen R, Zhang L, Hu H, Wu J, Zheng H et al (2018) Plasma asprosin levels are associated with glucose metabolism, lipid, and sex hormone profiles in females with metabolic-related diseases. Mediat Inflamm 2018:1-12. https://doi. org/10.1155/2018/7375294

Mauvais-Jarvis F, Clegg DJ, Hevener AL (2013) The role of estrogens in control of energy balance and glucose homeostasis. Endocr Rev 34(3):309-338. https://doi.org/10.1210/er.2012-1055

Muthu ML, Reinhardt DP (2020) Fibrillin-1 and fibrillin-1-derived asprosin in adipose tissue function and metabolic disorders. J Cell Commun Signal. https://doi.org/10.1007/s12079-020-00566-3

Reed BG, Carr BR (2000) The normal menstrual cycle and the control of ovulation. Endotext, pp 1-16. Retrieved from http://www.ncbi. nlm.nih.gov/pubmed/25905282

Romere C, Duerrschmid C, Bournat J, Constable P, Jain M, Xia F, Chopra AR et al (2016) Asprosin, a fasting-induced glucogenic 
protein hormone. Cell 165(3):566-579. https://doi.org/10.1016/j. cell.2016.02.063

Salpeter SR, Walsh JME, Ormiston TM, Greyber E, Buckley NS, Salpeter EE (2006) Meta-analysis: effect of hormone-replacement therapy on components of the metabolic syndrome in postmenopausal women. Diabetes Obes Metab 8(5):538-554. https://doi. org/10.1111/j.1463-1326.2005.00545.x

Sims ST, Heather AK (2018) Myths and methodologies: reducing scientific design ambiguity in studies comparing sexes and/or menstrual cycle phases. Exp Physiol 103(10):1309-1317. https://doi. org/10.1113/EP086797

Stubbins RE, Holcomb VB, Hong J, Núñez NP (2012) Estrogen modulates abdominal adiposity and protects female mice from obesity and impaired glucose tolerance. Eur J Nutr 51(7):861-870. https ://doi.org/10.1007/s00394-011-0266-4

Ugur K, Aydin S (2019) Saliva and blood asprosin hormone concentration associated with obesity. Int J Endocrinol 2019:1-8. https:// doi.org/10.1155/2019/2521096

United Nation Department of Economic and Social Affairs Population Divison (2015) Trends in contraceptive use Worldwide
2015. Contraception. https://doi.org/10.1016/j.contracept ion.2012.08.029

Wiecek M, Szymura J, Maciejczyk M, Kantorowicz M, Szygula Z (2018) Acute anaerobic exercise affects the secretion of asprosin, irisin, and other cytokines - a comparison between sexes. Front Physiol 9:1-12. https://doi.org/10.3389/fphys.2018.01782

Wilsterman K, Pepper A, Bentley GE (2017) Low glucose availability stimulates progesterone production by mouse ovaries in vitro. $\mathbf{J}$ Exp Biol 220(24):4583-4588. https://doi.org/10.1242/jeb.164384

Yuan M, Li W, Zhu Y, Yu B, Wu J (2020) Asprosin: a novel player in metabolic diseases. Front Endocrinol 11:1-7. https://doi. org/10.3389/fendo.2020.00064

Publisher's Note Springer Nature remains neutral with regard to jurisdictional claims in published maps and institutional affiliations. 